\title{
New analytical solutions of the space fractional KdV equation in terms of Jacobi elliptic functions
}

\author{
Aysegul Dascioglu, Sevil Culha, Dilek Varol Bayram \\ Department of Mathematics, Faculty of Science and Arts, Pamukkale University, Denizli, Turkey
}

Received: 12 September 2017, Accepted: 23 October 2017

Published online: 24 December 2017.

\begin{abstract}
In this study, new families of analytical exact solutions of the space fractional Korteweg-de Vries (KdV) equation are presented. Here, the fractional derivative is considered in conformable sense. By utilizing the Jacobi elliptic function expansion method, the solutions are obtained in general form containing the hyperbolic, trigonometric, and rational functions. Also, the complex valued solutions are obtained and some solutions of this equation are demonstrated.
\end{abstract}

Keywords: Jacobi elliptic function, fractional differential equation, KdV equation.

\section{Introduction}

It is well known that the Korteweg-de Vries (KdV) equation arises as a model for one-dimensional long wavelength surface waves propagating in weakly nonlinear dispersive media, as well as the evolution of weakly nonlinear ion acoustic waves in plasmas. So far, various methods are used for solving different types of fractional KdV equations. These methods can be listed as Adomian decomposition method [1,2], variational iteration method [2], homotopy perturbation method [3-7], differential transform method [8], modified direct algebraic method [9], $\left(G^{\prime} / G\right)$ - expansion method [10], improved $\left(G^{\prime} / G\right)$ - expansion method [10], homotopy perturbation transform method [11], function expansion method [12].

In this study, we consider the space fractional Korteweg-de Vries equation

$$
u_{t}+u D_{x}^{\alpha} u+\beta D_{x}^{\alpha} D_{x}^{\alpha} D_{x}^{\alpha} u=0, \quad 0<\alpha \leq 1
$$

Here, $D_{x}^{\alpha}$ means conformable fractional derivative of function $u(x, t)$ with respect to $x$, and $\beta$ is a constant. When $\alpha=1$ the equation (1) becomes the known KdV equation of integer order.

For finding the analytical solutions of equation (1), we present an expansion method using the Jacobi elliptic functions. The main idea in this method is to find the solutions $u(\xi)$ in the form

$$
u(\xi)=\sum_{j=0}^{N} a_{j} F^{j}(\xi)
$$

where $N, a_{j}(j=0,1,2, \ldots, N)$ are constants to be determined later and $F(\xi)$ is the solution of the Jacobi elliptic equation

$$
\left(F^{\prime}\right)^{2}(\xi)=P+Q F^{2}(\xi)+R F^{4}(\xi)
$$

Here, $F^{\prime}=\frac{d F}{d \xi}, \xi=\xi(t, x)$ and $P, Q, R$ are constants. Eq.(3) has Jacobi elliptic function solutions for the different values of $P, Q$ and $R$. The Jacobi elliptic functions corresponding to some values of $P, Q$ and $R$ can be found in [13]. 


\section{Preliminaries}

The basic Jacobian elliptic functions are

$$
\operatorname{sn} \xi=\operatorname{sn}(\xi ; m), \operatorname{cn} \xi=\operatorname{cn}(\xi ; m), \operatorname{dn} \xi=\operatorname{dn}(\xi ; m)
$$

where $m(0<m<1)$ is the modulus of the elliptic function. When $m \rightarrow 0$ and $m \rightarrow 1$ Jacobi elliptic functions turn into trigonometric and hyperbolic functions [14]. There are different definitions of the fractional derivative such as Grunwald-Letnikov, Riemann-Liouville and Caputo [15-17]. However, there are some setbacks of these definitions [18]. To overcome these difficulties, Khalil et al. had given a new definition of fractional derivative named as conformable fractional derivative. This is the simplest definition to recognize the fractional derivative since it is the most familiar one to the definition of the usual derivative. Therefore, the $\mathrm{KdV}$ equation is considered in conformable sense. The definition and the properties of the conformable derivative are as follows:

Definition 1. (Conformable Fractional Derivative) [ 18 ] Let $f:[0, \infty] \rightarrow R$ be a function. The $\alpha$-th order conformable fractional derivative of $f$ is defined by

$$
T_{\alpha}(f)(t)=\lim _{\varepsilon \rightarrow 0} \frac{f\left(t+\varepsilon t^{1-\alpha}\right)-f(t)}{\varepsilon}, \quad t>0, \quad \alpha \in(0,1) .
$$

If $f$ is $\alpha$-differentiable in some $(0, \alpha), \alpha>0$ and $\lim _{t \rightarrow 0^{+}} f^{(\alpha)}(t)$ exists, then we define $f^{(\alpha)}(0)=\lim _{t \rightarrow 0^{+}} f^{(\alpha)}(t)$.

Theorem 1. [18] Let $\alpha \in(0,1]$ and suppose $f$, $g$ are $\alpha$-differentiable at point $t>0$. Then, the following are satisfied:

(i) $T_{\alpha}(c f+d g)=c T_{\alpha}(f)+d T_{\alpha}(g) \forall c, d \in R$.

(ii) $T_{\alpha}\left(t^{p}\right)=p t^{p-\alpha} \forall p \in R$.

(iii) $T_{\alpha}(\lambda)=0$ for all constant functions $f(t)=\lambda$.

(iv) $T_{\alpha}(f g)=f T_{\alpha}(g)+g T_{\alpha}(f)$.

(v) $T_{\alpha}\left(\frac{f}{g}\right)=\frac{g T_{\alpha}(f)-f T_{\alpha}(g)}{g^{2}}$.

(vi) If, in addition, $f$ is differentiable, then $T_{\alpha}(f)(t)=t^{1-\alpha} \frac{d f}{d t}$.

Theorem 2. (Chain Rule) [18] Assume $f, g:(0, \infty) \rightarrow R$ be $\alpha$-differentiable and for all $t$ with $t \neq 0$ and $g(t) \neq 0$ we have

$$
T_{\alpha}(h)(t)=T_{\alpha}(f)(g(t)) \cdot T_{\alpha}(g)(t) \cdot g(t)^{\alpha-1}
$$

If $t=0$ we have

$$
T_{\alpha}(h)(0)=\lim _{t \rightarrow 0} T_{\alpha}(f)(g(t)) \cdot T_{\alpha}(g)(t) \cdot g(t)^{\alpha-1} .
$$

\section{Solutions to the conformable space-fractional KdV equation}

Let us consider the conformable space-fractional $\mathrm{KdV}$ equation (1). Changing of the variables in the equation as

$$
u=u(\xi), \quad \xi=k \frac{x^{\alpha}}{\alpha}+l t
$$

yields an ordinary differential equation (ODE) for $u(\xi)$

$$
l \frac{d u}{d \xi}+k u \frac{d u}{d \xi}+\beta k^{3} \frac{d^{3} u}{d \xi^{3}}=0
$$


where $k$ and $l$ are the arbitrary constants. Solving this nonlinear ordinary differential equation, the integer $N$ in expression (2) can be determined by balancing the highest order linear term

$$
O\left(u \frac{d^{3} u}{d \xi^{3}}\right)=N+3
$$

and the highest order nonlinear term

$$
O\left(u \frac{d u}{d \xi}\right)=2 N+1
$$

in (5). As the result of this balancing, we obtain $N=2$, thus the solution of Eq. (1)can be expressed as

$$
u(\xi)=\sum_{j=0}^{2} a_{j} F^{j}(\xi)=a_{0}+a_{1} F+a_{2} F^{2} .
$$

By differentiating Eq. (8) three times, we get

$$
\begin{gathered}
u^{\prime}(\xi)=a_{1} F^{\prime}+2 a_{2} F F^{\prime}, \\
u^{\prime \prime \prime}(\xi)=a_{1} F^{\prime \prime \prime}+6 a_{2} F^{\prime} F^{\prime \prime \prime}+2 a_{2} F F^{\prime \prime}
\end{gathered}
$$

and using Eq. (3), we have

$$
\begin{gathered}
F^{\prime \prime}=2 R F^{3}+Q F, \\
F^{\prime \prime \prime}=6 R F^{2} F^{\prime}+Q F^{\prime} .
\end{gathered}
$$

Substituting the equations (11) and (12) into Eq. (10) yields

$$
u^{\prime \prime \prime}=a_{1} Q F^{\prime}+6 a_{1} R F^{2} F^{\prime}+8 a_{2} Q F F^{\prime}+24 a_{2} R F^{3} F^{\prime} .
$$

Finally, substituting equations (9) and (13) into Eq. (5) and setting each coefficient of $\mathrm{F}$ to be zero, the following equations system arises

$$
\begin{gathered}
l a_{1}+k a_{0} a_{1}+\beta k^{3} a_{1} Q=0 \\
2 l a_{2}+2 k a_{0} a_{2}+k a_{1}^{2}+8 \beta k^{3} a_{2} Q=0 \\
3 k a_{1} a_{2}+k a_{1}^{2}+6 \beta k^{3} a_{1} R=0 \\
2 k a_{2}^{2}+24 \beta k^{3} a_{2} R=0 .
\end{gathered}
$$

Solving this system, we get

$$
a_{0}=B+4 A Q, \quad a_{1}=0, \quad a_{3}=12 A R
$$

such that $A=-\beta k^{2}, B=-(l / k)$. Hence, the solution of the Eq. (5) is

$$
u=B+4 A Q+12 A R F^{2} .
$$

For the different values of the constants $Q$ and $R$, we get the solutions of eq. (5) as in the following Table 1. By substituting $\xi$ defined in (4), we obtain the exact solutions of the KdV equation (1). 
Table 1: Solutions of Eq. (5) for the special values of $P, Q$ and $R$.

\begin{tabular}{|c|c|c|c|c|}
\hline & $\mathbf{P}$ & Q & $\mathbf{R}$ & Solutions \\
\hline \multirow[b]{2}{*}{1} & \multirow{2}{*}{1} & \multirow{2}{*}{$-\left(1+m^{2}\right)$} & \multirow{2}{*}{$m^{2}$} & $u_{1,1}=B-4 A\left(1+m^{2}\right)+12 A m^{2} \operatorname{sn}^{2} \xi$ \\
\hline & & & & $u_{1,2}=B-4 A\left(1+m^{2}\right)+12 A m^{2} c^{2} \xi$ \\
\hline 2 & $1-m^{2}$ & $2 m^{2}-1$ & $-m^{2}$ & $u_{2}=B+4 A\left(2 m^{2}-1\right)-12 A m^{2} \mathrm{cn}^{2} \xi$ \\
\hline 3 & $m^{2}-1$ & $2-m^{2}$ & -1 & $u_{3}=B+4 A\left(2-m^{2}\right)-12 A \operatorname{dn}^{2} \xi$ \\
\hline \multirow[b]{2}{*}{4} & \multirow{2}{*}{$m^{2}$} & \multirow[b]{2}{*}{$-\left(1+m^{2}\right)$} & \multirow[b]{2}{*}{1} & $u_{4,1}=B-4 A\left(1+m^{2}\right)+12 A \mathrm{~ns}^{2} \xi$ \\
\hline & & & & $u_{4,2}=B-4 A\left(1+m^{2}\right)+12 A \mathrm{dc}^{2} \xi$ \\
\hline 5 & $-m^{2}$ & $2 m^{2}-1$ & $1-m^{2}$ & $u_{5}=B+4 A\left(2 m^{2}-1\right)+12 A\left(1-m^{2}\right) n c^{2} \xi$ \\
\hline 6 & -1 & $2-m^{2}$ & $m^{2}-1$ & $u_{6}=B+4 A\left(2-m^{2}\right)+12 A\left(m^{2}-1\right) \mathrm{nd}^{2} \xi$ \\
\hline 7 & 1 & $2-m^{2}$ & $1-m^{2}$ & $u_{7}=B+4 A\left(2-m^{2}\right)+12 A\left(1-m^{2}\right) s^{2} \xi$ \\
\hline 8 & 1 & $2 m^{2}-1$ & $-m^{2}+m^{4}$ & $u_{8}=B+4 A\left(2 m^{2}-1\right)+12 A\left(-m^{2}+m^{4}\right) s d^{2} \xi$ \\
\hline 9 & $1-m^{2}$ & $2-m^{2}$ & 1 & $u_{9}=B+4 A\left(2-m^{2}\right)+12 A \mathrm{cs}^{2} \xi$ \\
\hline 10 & $-m^{2}+m^{4}$ & $2 m^{2}-1$ & 1 & $u_{10}=B+4 A\left(2 m^{2}-1\right)+12 A \mathrm{ds}^{2} \xi$ \\
\hline 11 & $-\frac{\left(1-m^{2}\right)^{2}}{4}$ & $\frac{1+m^{2}}{2}$ & $-\frac{1}{4}$ & $u_{11}=B+2 A\left(1+m^{2}\right)-3 A(m \operatorname{cn} \xi \mp \operatorname{dn} \xi)^{2}$ \\
\hline 12 & $\frac{1}{4}$ & $\frac{-2 m^{2}+1}{2}$ & $\frac{1}{4}$ & $u_{12}=B-2 A\left(2 m^{2}-1\right)+3 A(\mathrm{~ns} \xi \mp \operatorname{cs} \xi)^{2}$ \\
\hline 13 & $\frac{1-m^{2}}{4}$ & $\frac{1+m^{2}}{2}$ & $\frac{1-m^{2}}{4}$ & $u_{13}=B+2 A\left(m^{2}+1\right)+3 A\left(1-m^{2}\right)(\mathrm{nc} \xi \mp s c \xi)^{2}$ \\
\hline 14 & $\frac{m^{4}}{4}$ & $\frac{m^{2}-2}{2}$ & $\frac{1}{4}$ & $u_{14}=B+2 A\left(m^{2}-2\right)+3 A(\mathrm{~ns} \xi \mp \mathrm{d} s \xi)^{2}$ \\
\hline \multirow{2}{*}{15} & \multirow{2}{*}{$\frac{m^{2}}{4}$} & \multirow{2}{*}{$\frac{m^{2}-2}{2}$} & \multirow{2}{*}{$\frac{m^{2}}{4}$} & $u_{15,1}=B+2 A\left(m^{2}-2\right)+3 A m^{2}(\operatorname{sn} \xi \mp i \mathrm{cn} \xi)^{2}$ \\
\hline & & & & $u_{15,2}=B+2 A\left(m^{2}-2\right)+3 A m^{2} \frac{\operatorname{dn}^{2} \xi}{1-m^{2} \operatorname{sn} \xi \mp \operatorname{cn} \xi}$ \\
\hline \multirow[b]{2}{*}{16} & \multirow{2}{*}{$\frac{1}{4}$} & \multirow{2}{*}{$\frac{1-2 m^{2}}{2}$} & \multirow{2}{*}{$\frac{1}{4}$} & $u_{16,1}=B+2 A\left(1-2 m^{2}\right)+3 A(m \operatorname{cn} \xi \mp i \mathrm{dn} \xi)^{2}$ \\
\hline & & & & $u_{16,2}=B+2 A\left(m^{2}-2\right)+3 A\left(\frac{\operatorname{sn} \xi}{1 \mp \operatorname{cn} \xi}\right)^{2}$ \\
\hline 17 & $\frac{1}{4}$ & $\frac{m^{2}-2}{2}$ & $\frac{m^{2}}{4}$ & $u_{17}=B+2 A\left(m^{2}-2\right)+3 A m^{2}\left(\frac{\operatorname{sn} \xi}{1 \mp \operatorname{dn} \xi}\right)^{2}$ \\
\hline 18 & $\frac{m^{2}-1}{4}$ & $\frac{1+m^{2}}{2}$ & $\frac{m^{2}-1}{4}$ & $u_{18}=B+2 A\left(1+m^{2}\right)+3 A\left(m^{2}-1\right)\left(\frac{\operatorname{dn} \xi}{1 \mp m s n \xi}\right)^{2}$ \\
\hline 19 & $\frac{1-m^{2}}{4}$ & $\frac{1+m^{2}}{2}$ & $\frac{1-m^{2}}{4}$ & $u_{19}=B+2 A\left(1+m^{2}\right)+3 A\left(1-m^{2}\right)\left(\frac{\mathrm{cn} \xi}{1 \mp \operatorname{sn} \xi}\right)^{2}$ \\
\hline 20 & $\frac{1}{4}$ & $\frac{1+m^{2}}{2}$ & $\frac{\left(1-m^{2}\right)^{2}}{4}$ & $u_{20}=B+2 A\left(1+m^{2}\right)+3 A\left(1-m^{2}\right)^{2}\left(\frac{\operatorname{sn} \xi}{\operatorname{dn} \xi \mp \operatorname{cn} \xi}\right)^{2}$ \\
\hline 21 & $\frac{1}{4}$ & $\frac{m^{2}-2}{2}$ & $\frac{m^{2}}{4}$ & $u_{21}=B+2 A\left(m^{2}-2\right)+3 A m^{2} \frac{\mathrm{cn}^{2} \xi}{1-m^{2} \mp \operatorname{dn} \xi}$ \\
\hline
\end{tabular}

Considering the solutions in Table 1 and the behavior of the Jacobi elliptic functions for $m \rightarrow 0$ and $m \rightarrow 1$, the elementary function solutions can be given at Table 2 . 
Table 2: The behavior of the solutions in the Table 1 when $m \rightarrow 0$ and $m \rightarrow 1$.

\begin{tabular}{|c|c|c|c|}
\hline & Solutions & $m \rightarrow 0$ & $m \rightarrow 1$ \\
\hline \multirow{2}{*}{1} & $u_{1,1}$ & $B-4 A$ & $B-8 A+12 A \tanh ^{2} \xi$ \\
\hline & $u_{1,2}$ & $B-4 A$ & $B+4 A$ \\
\hline 2 & $u_{2}$ & $B-4 A$ & $B+4 A-12 A \operatorname{sech}^{2} \xi$ \\
\hline 3 & $u_{3}$ & $B-4 A$ & $B+4 A-12 A \operatorname{sech}^{2} \xi$ \\
\hline \multirow{2}{*}{4} & $u_{4,1}$ & $B-4 A+12 A \csc ^{2} \xi$ & $B-8 A+12 A \operatorname{coth}^{2} \xi$ \\
\hline & $u_{4,2}$ & $B-4 A+12 A \sec ^{2} \xi$ & $B+4 A$ \\
\hline 5 & $u_{5}$ & $B-4 A+12 A \sec ^{2} \xi$ & $B+4 A$ \\
\hline 6 & $u_{6}$ & $B-4 A$ & $B+4 A$ \\
\hline 7 & $u_{7}$ & $B+8 A+12 A \tan ^{2} \xi$ & $B+4 A$ \\
\hline 8 & $u_{8}$ & $B-4 A$ & $B+4 A$ \\
\hline 9 & $u_{9}$ & $B+8 A+12 A \cot ^{2} \xi$ & $B+4 A+12 A \operatorname{csch}^{2} \xi$ \\
\hline 10 & $u_{10}$ & $B-4 A+12 A \csc ^{2} \xi$ & $B+4 A+12 A \operatorname{csch}^{2} \xi$ \\
\hline 11 & $u_{11}$ & $B-A$ & $B+4 A$ \\
\hline 12 & $u_{12}$ & $B+2 A+3 A(\csc \xi \mp \cot \xi)^{2}$ & $B-2 A+3 A(\operatorname{csch} \xi \mp \operatorname{coth} \xi)^{2}$ \\
\hline 13 & $u_{13}$ & $B+2 A+3 A(\sec \xi \mp \tan \xi)^{2}$ & $\begin{array}{l}B+4 A \\
B+4 A-12 A \operatorname{sech}^{2} \xi\end{array}$ \\
\hline 14 & $u_{14}$ & $\begin{array}{l}B-4 A \\
B-4 A+12 A \csc ^{2} \xi\end{array}$ & $B-2 A+3 A(\operatorname{coth} \xi \mp \operatorname{csch} \xi)^{2}$ \\
\hline \multirow[b]{2}{*}{15} & $u_{15,1}$ & $B-4 A$ & $B-2 A+3 A(\tanh \xi \mp i \operatorname{sech} \xi)^{2}$ \\
\hline & $u_{15,2}$ & $B-4 A$ & $B-2 A+3 A \frac{\operatorname{sech}^{2} \xi}{1-\tanh \xi \bar{T} \operatorname{sech} \xi}$ \\
\hline \multirow[b]{2}{*}{16} & $u_{16,1}$ & $B-A$ & $B-2 A+3 A((1 \mp i) \operatorname{sech} \xi)^{2}$ \\
\hline & $u_{16,2}$ & $B-4 A+3 A\left(\frac{\sin \xi}{1 \mp \cos \xi}\right)^{2}$ & $B-2 A+3 A\left(\frac{\sinh \xi}{\cosh \xi \bar{\mp} 1}\right)^{2}$ \\
\hline 17 & $u_{17}$ & $B-4 A$ & $B-2 A+3 A\left(\frac{\sinh \xi}{\cosh \xi \mp 1}\right)^{2}$ \\
\hline 18 & $u_{18}$ & $B-A$ & $B+4 A$ \\
\hline 19 & $u_{19}$ & $B+2 A+3 A\left(\frac{\cos \xi}{1 \bar{\mp} \sin \xi}\right)^{2}$ & $B+4 A$ \\
\hline 20 & $u_{20}$ & $B+2 A+3 A\left(\frac{\sin \xi}{1 \mp \cos \xi}\right)^{2}$ & $B+4 A$ \\
\hline 21 & $u_{21}$ & $B-4 A$ & $B-2 A \mp 3 A \operatorname{sech} \xi$ \\
\hline
\end{tabular}

\section{Demonstrations of some solutions}

In this section, we give 8 figures demonstrating two solutions from Table 1 and two solutions from Table 2 in both $2 \mathrm{D}$ and 3D plots. In all figures, the solutions are considered for $k=l=\beta=1$. All graphics in figures are drawn by the aid of 
Mathematica. Firstly, let us reconsider solution $u_{2}$ in Table 1,

$$
u_{2}=3-8 m^{2}+12 m^{2} \mathrm{cn}^{2} \xi
$$

We demonstrate the above solution for $0 \leq \xi \leq 5$ when $0 \leq m \leq 1$ and $m=0.5$ in Figure 1 and in Figure 2, respectively.

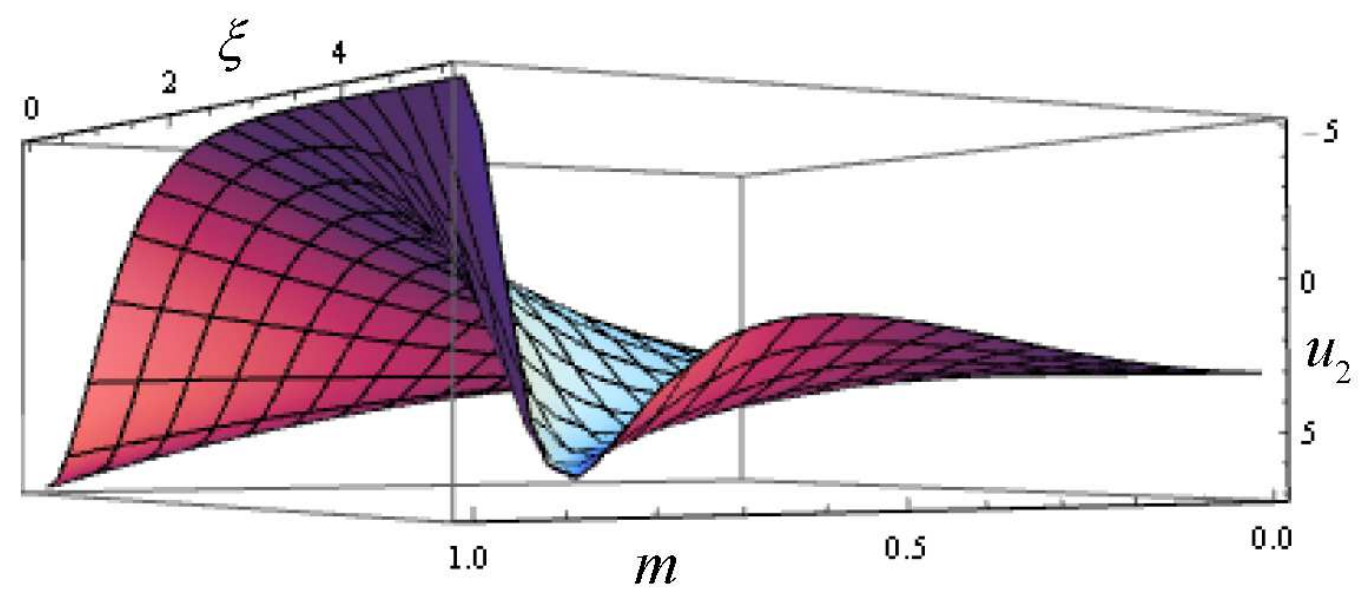

Fig. 1: 3D plot of the obtained solution $u_{2}(\xi)$ when $0 \leq m \leq 1$

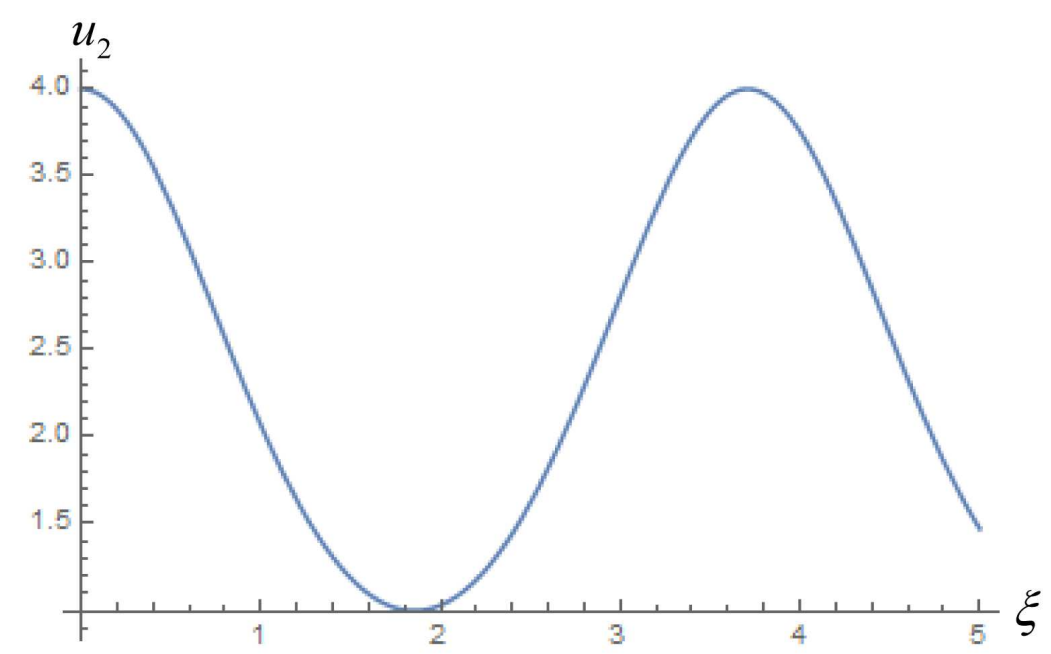

Fig. 2: 2D plot of the solution $u_{2}(\xi)$ when $m=0.5$

When $m \rightarrow 0, u_{2}=3$ and when $m \rightarrow 1, u_{2}=-5+12 \operatorname{sech}^{2}(2 \sqrt{x}+t)$ for $\alpha=0.5$. We demonstrate the second case for $0 \leq x \leq 4$ when $0 \leq t \leq 1$ and at $t=1$ in Figure 3 and in Figure 4, respectively. 


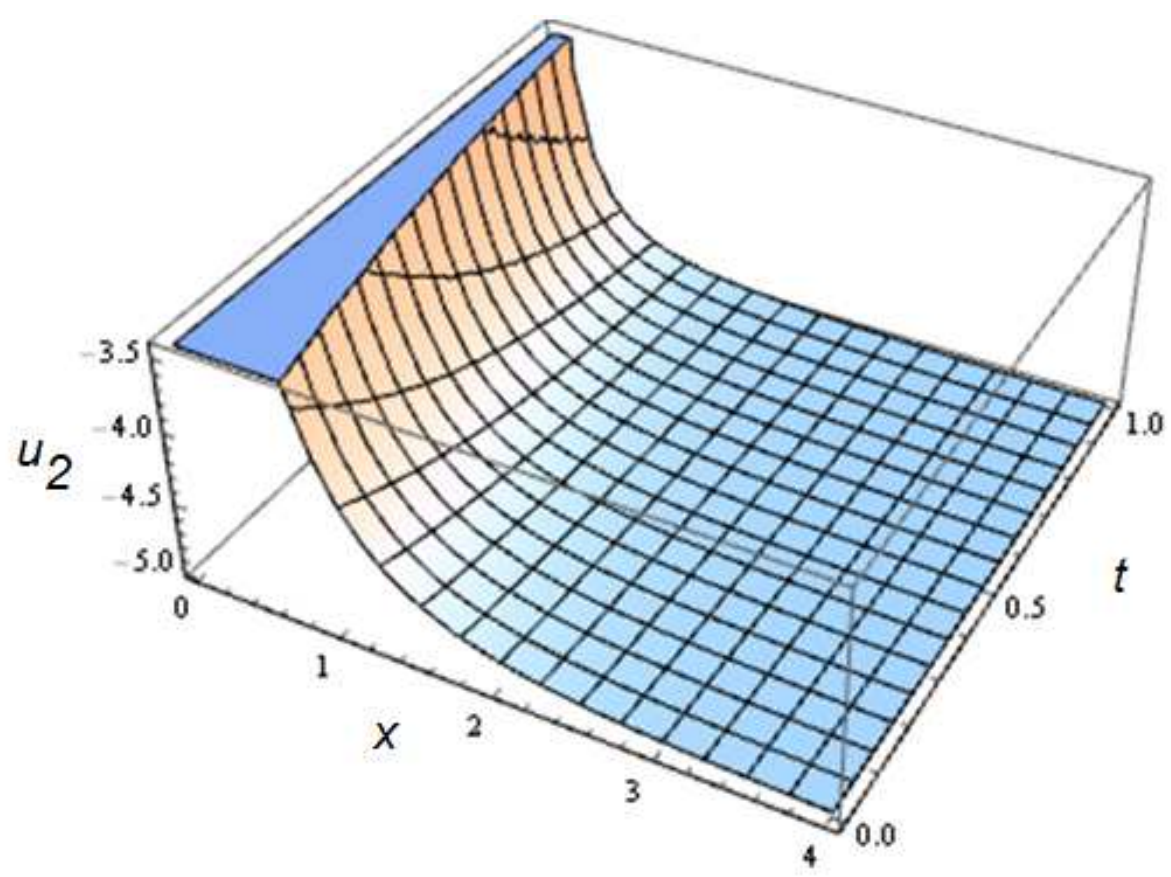

Fig. 3: 3D plot of the obtained solution $u_{2}(x, t)$ when $m \rightarrow 1$.

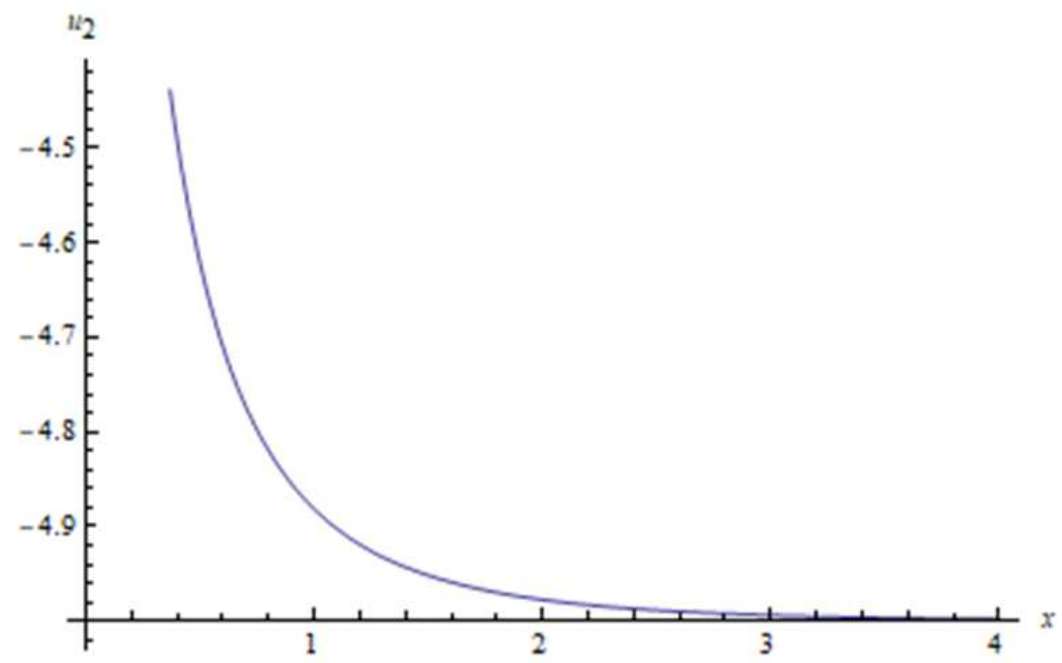

Fig. 4: 2D plot of the solution $u_{2}(x, 1)$ when $m \rightarrow 1$.

Secondly, we analyze the solution $u_{4,2}$ in Table 1

$$
u_{4,2}=3+4 m^{2}-12 \mathrm{dc}^{2} \xi
$$

We demonstrate the considered solution for $1 \leq \xi \leq 4$ when $0 \leq m \leq 1$ and $m=0.5$ in Figure 5 and in Figure 6 , respectively. 


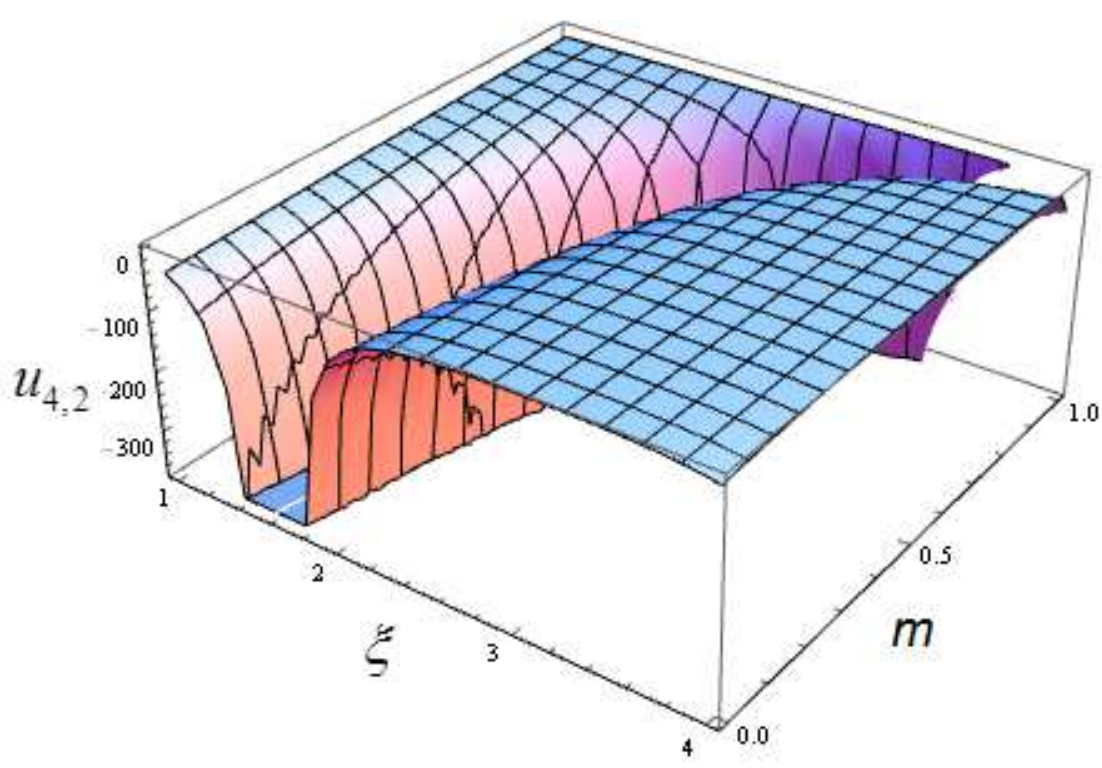

Fig. 5: 3D plot of the obtained solution $u_{4}, 2(\xi)$ when $0 \leq m \leq 1$.

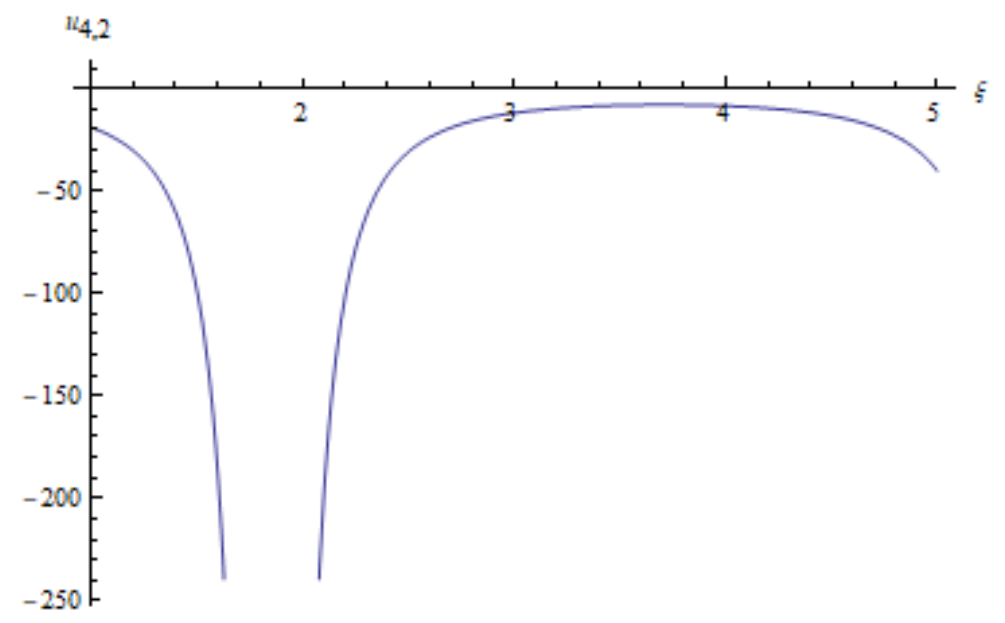

Fig. 6: $2 \mathrm{D}$ plot of the obtained solution $u_{4}, 2(\xi)$ when $m=0.5$.

When $m \rightarrow 1, u_{4,2}=-5$ and when $m \rightarrow 0, u_{4,2}=3-12 \sec ^{2}(2 \sqrt{x}+t)$ for $\alpha=0.5$. We demonstrate the second case for $1 \leq x \leq 4$ when $0 \leq t \leq 1$ and at $t=0.5$ in Figure 7 and in Figure 8, respectively. 


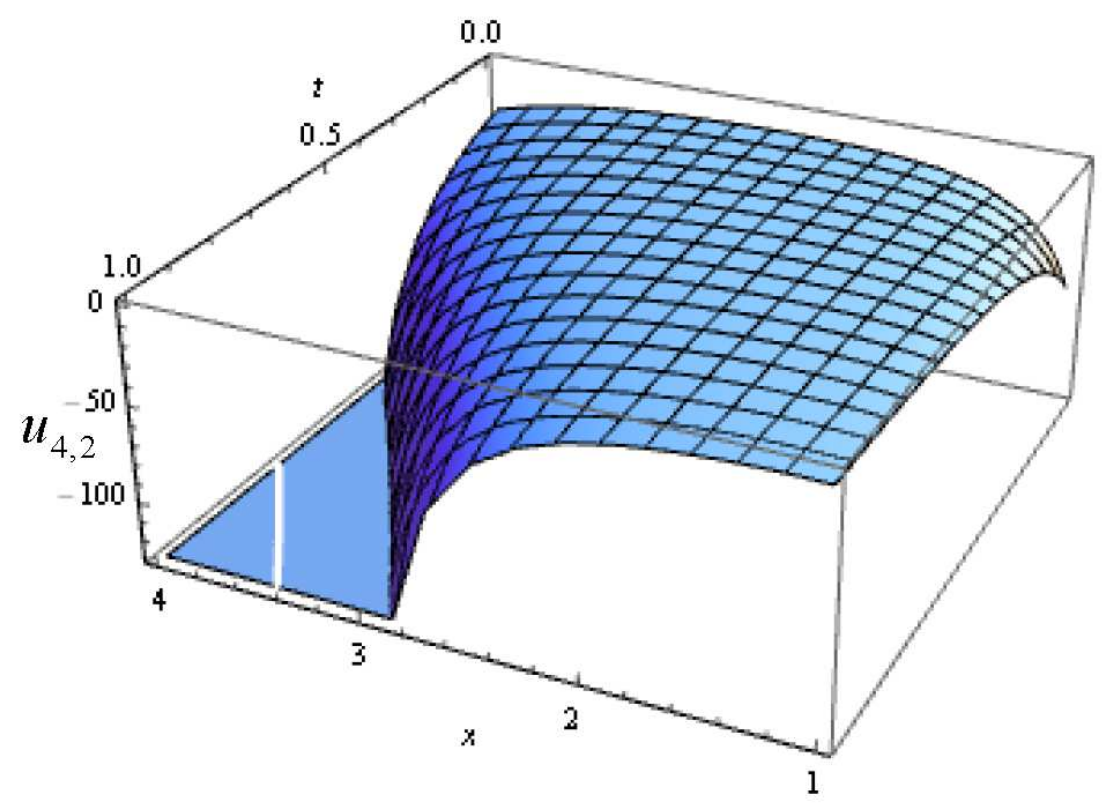

Fig. 7: 3D plot of the obtained solution $u_{4,2}(x, t)$ when $m \rightarrow 0$.

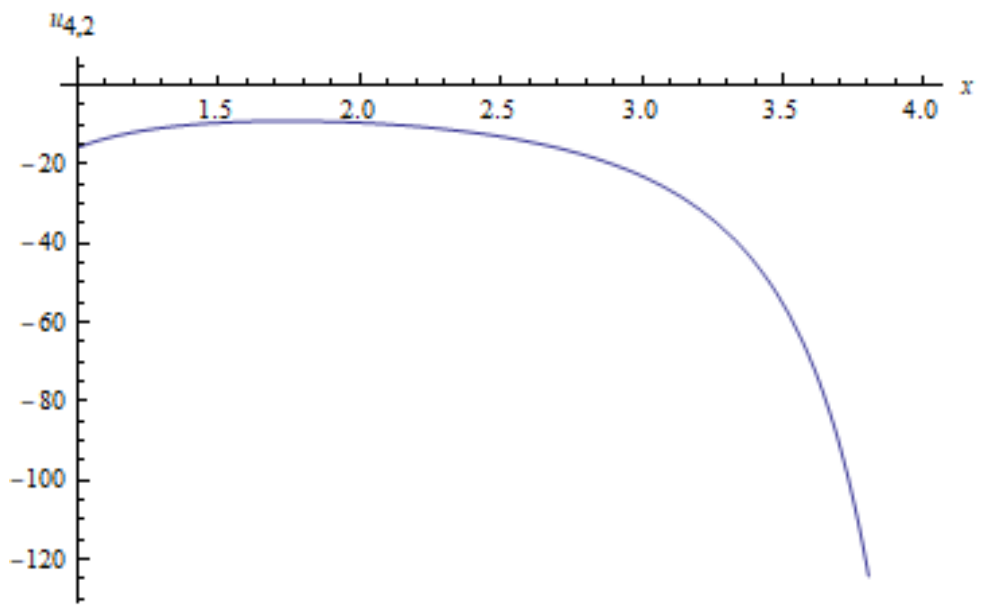

Fig. 8: 2 D plot of the obtained solution $u_{4,2}(x, 0.5)$ when $m \rightarrow 0$.

\section{Conclusion}

In this study, the Jacobi elliptic function expansion method is used to obtain the exact solutions of the conformable spacefractional KdV equation. This method obtains the results directly, quickly and needs simple algorithms in programming. By this powerful method, the solutions are found in hyperbolic, trigonometric, and rational function form involving some parameters; thus, by this single method we cover the solutions of several different methods at the same time. Additionally, by using conformable fractional derivative definition, fractional wave equations can be solved easily rather than the other definitions. 


\section{Acknowledgments}

This work is supported by the Scientific Research Project Coordination Unit of Pamukkale University with number 2017FEBE-00455.

\section{Competing interests}

The authors declare that they have no competing interests.

\section{Authors' contributions}

All authors have contributed to all parts of the article. All authors read and approved the final manuscript.

\section{References}

[1] S. Momani, An explicit and numerical solutions of the fractional KdV equation, Mathematics and Computers in Simulation 70 (2005) $110-118$.

[2] Z. M. Odibat, Exact solitary solutions for variants of the KdV equations with fractional time derivatives, Chaos, Solitons and Fractals 40 (2009) 1264-1270.

[3] Q. Wang, Homotopy perturbation method for fractional KdV equation, Applied Mathematics and Computation 190 (2007) 17951802.

[4] Z. M. Odibat, Compact and noncompact structures for nonlinear fractional evolution equations, Physics Letters A 372 (2008) 1219-1227.

[5] Z. M. Odibat, Compact structures in a class of nonlinearly dispersive equations with time-fractional derivatives, Applied Mathematics and Computation 205 (2008) 273-280.

[6] O. Abdulaziz, I. Hashim, E.S. Ismail, Approximate analytical solution to fractional modified KdV equations, Mathematical and Computer Modelling 49 (2009) 136-145.

[7] Z. Odibat, S. Momani, The variational iteration method: An efficient scheme for handling fractional partial differential equations in fluid mechanics, Computers and Mathematics with Applications 58 (2009) 2199-2208.

[8] M. Kurulay, M. Bayram, Approximate analytical solution for the fractional modified KdV by differential transform method, Commun Nonlinear Sci Numer Simulat 15 (2010) 1777-1782.

[9] M. Younis, H. Rehman, M. Iftikhar, Travelling wave solutions to some time-space nonlinear evolution equations, Applied Mathematics and Computation 249 (2014) 81-88.

[10] S. Sahoo, S. S. Ray, Solitary wave solutions for time fractional third order modified KdV equation using two reliable techniques $\left(G^{\prime} / G\right)$-expansion method and improved $\left(G^{\prime} / G\right)$-expansion method, Physica A 448 (2016) 265-282.

[11] S. Arshad, A. Sohail, K. Maqbool, Nonlinear shallow water waves: A fractional order Approach, Alexandria Engineering Journal (2016) 55, 525-532.

[12] W. Rui, Applications of homogenous balanced principle on investigating exact solutions to a series of time fractional nonlinear PDEs, Commun Nonlinear Sci Numer Simulat 47 (2017) 253-266.

[13] L. Hua-Mei, New exact solutions of nonlinear Gross-Pitaevskii equation with weak bias magnetic and time-dependent laser fields, Chinese Physics 14 (2) (2005) 251-256.

[14] J. V Armitage ve W. F. Eberlein, Elliptic Functions, New York: Cambridge University Press, 2006.

[15] K.S. Miller, An Introduction to Fractional Calculus and Fractional Differential Equations, J. Wiley and Sons, New York, 1993.

[16] A. Kilbas, H. Srivastava, J. Trujillo, Theory and Applications of Fractional Differential Equations, in: Math. Studies., NorthHolland, New York, 2006.

[17] I. Podlubny, Fractional Differential Equations, Academic Press, USA, 1999.

[18] R. Khalil, M. A. Horani, A. Yousef and M. Sababheh, A new definition of fractional derivative, Journal of Computational and Applied Mathematics 264 (2014) 65-70.

[19] T. Abdeljawad, On conformable fractional calculus, Journal of Computational and Applied Mathematics 279 (2015) 57-66. 